\title{
Gluten Immunogenic Peptides (GIP) Point-of-Care Urine Test in Coeliac Disease Follow-up before and during the COVID-I9 Lockdown in Italy
}

\author{
Carolina Ciacci $\mathbb{D}^{\prime}$ \\ Mario Gagliardi (D) \\ Monica Siniscalchi (iD) \\ Monica Ruotolo' \\ Antonella Santonicola (D) \\ Najla Hajji (iD) \\ Fabiana Zingone (iD) ${ }^{2}$ \\ 'Celiac Center at Department of \\ Medicine, Surgery, and Dentistry, Scuola \\ Medica Salernitana, University of Salerno, \\ Salerno, Italy; ${ }^{2}$ Gastroenterology Unit, \\ Department of Surgery, Oncology and \\ Gastroenterology, University of Padua, \\ Padua, Italy
}

Introduction: Celiac disease $(\mathrm{CeD})$ diagnosis has worldwide shared protocols. Conversely, follow-up of patients is still an object of study. Gluten immunogenic peptide detection in the urine (GIP) appears to be a new and efficient method for dietary gluten control of patients. The present study aims to assess the clinical usefulness of the GIP point-of-care urine test in the follow-up of symptomatic and asymptomatic patients with $\mathrm{CeD}$ before and during the COVID-19 lockdown in Italy.

Methods: Thirty adult CeD patients on a gluten-free diet (GFD) were enrolled before and during the COVID-19 lockdown through follow-up visits or remote consultation. Patients underwent anthropometrical evaluation, dietetic interview, and State-Trait Anxiety Inventory (STAI). Then, two groups were formed: symptomatic and worried about gluten contamination. Each patient received 5 GIP point-of-care tests to perform a maximum of 5 times in the following 5 weeks in case of symptoms or anxiety state due to hypothesized gluten contamination.

Results: Sixteen symptomatic patients and 14 patients with concerns related to gluten contamination were included. There were no differences in age, BMI, compliance to GFD and GIP positive tests between the two groups. Worried group showed a borderline higher level of anxiety than symptomatic group $(p=0.06)$, with a significant minor percentage of patients reporting "no or low anxiety" $(14.3 \%$ vs $50 \% \mathrm{p}=0.03)$. The symptomatic patients showed a higher rate of diarrhea than worried group $(25 \%$ vs $0 \%, \mathrm{p}=0.04)$. Gluten in urine samples was globally found in 8 out of 30 cases $(26.6 \%)$.

Conclusion: The GIP test is a tool that can be used as a point of care test to assess adequate compliance with GFD and reassure symptomatic CeD patients from the feeling of anxiety for gluten contamination, especially during the COVID-19 pandemic.

Keywords: GIP, POCT gluten contamination, GFD, anxiety, burden, celiac disease

\section{Introduction}

Coeliac disease $(\mathrm{CeD})$ is an immune-mediated disease caused by the ingestion of gluten in genetically predisposed individuals with an approximate prevalence of $1 \%$ and a female predominance. Any age can be affected by a variety of symptoms/ manifestations. Diagnostic testing, including serology and biopsy, should be performed on a gluten-containing diet. The treatment for CD is a long-life gluten-free diet (GFD). The aims during follow-up are the absence of symptoms and achieving mucosal healing. Dietary compliance can be evaluated through a specific questionnaire (ie, Biagi's questionnaire ${ }^{1}$ ) and evaluating antibodies anti-transglutaminase
Correspondence: Carolina Ciacci Celiac Center at Department of Medicine, Surgery, and Dentistry, Scuola Medica Salernitana, University of Salerno, Baronissi, Salerno, 8408I, Italy

Email cciacci@unisa.it 
IgA. However, serum antibodies have poor sensitivity for persistent villous atrophy and, therefore, for minimum gluten contamination. ${ }^{2}$

On the other hand, intestinal histology is highly sensitive, but it has the disadvantage of being invasive and not readily accepted by patients. Gluten immunogenic peptide detection in the urine (GIP) appears to be a new and efficient method for dietary gluten control of $\mathrm{CeD}$ patients. Several methods for the detection of GIP show high sensitivity and specificity. ${ }^{3,4}$ GIP seems to respond to the request of checking on the GFD adherence as a proxy of the intestinal mucosa status and the risk of CeD-related complications. $^{5-7}$ An observational, prospective, crosssectional study showed that $11 / 44(25 \%) \mathrm{CeD}$ patients returned at least one positive GIP test despite a strict GFD. ${ }^{6}$ However, studies assessing the usefulness of the GIP test in real life are still scanty.

At the beginning of the COVID-19 lockdown in Italy, on March 9, 2020, among the others, also CeD patients could not reach the General Practitioners (GPs) or the outpatients' clinic for regular care. Italian Government encouraged health-care givers to set up free remote care by all possible means, taking into account that Italian patients usually participate in their healthcare as they possess their clinical records and have regular spontaneous access also to the tertiary clinics.

The present study aims to assess the clinical usefulness of the GIP point-of-care urine test in the follow-up of symptomatic and asymptomatic patients with $\mathrm{CeD}$ before and during the COVID-19 lockdown in Italy.

\section{Materials and Methods}

We included all eligible adult patients visited at the Celiac Center of the University of Salerno from June 2019 to February 2020 (pre-Covid period); then, during the COVID-19 lockdown from March 16 to September 30, 2020, we enrolled patients who were attending remote consultations (Covid period). The study was approved by the Campania Sud Ethical Committee (protocol no.3-29.05.2019). All patients provided written informed consent for the publication of the case details and any accompanying images. This study was conducted in accordance with the Declaration of Helsinki.

The inclusion criteria were a well-established serology and histological diagnosis of $\mathrm{CeD}$ based on positive serology tests and concomitant duodenal biopsy showing villous atrophy (Marsh 3); self-reported adherence to the GFD for more than one year and the ability to collect and send by email/WhatsApp the results of GIP tests. The exclusion criteria were immunodeficiency, evident lapses in the GFD, positive serology (anti-TTG IgA antibodies), and lack of consent. During the recruitment visit, enrolled patients underwent anthropometry evaluation and dietetic interview with a Visual Analogue Scale to self-evaluate the quality of their diet (0 no adherence 10 full adherence). Then, the patients were stratified based on the reporting of any gastrointestinal or nongastrointestinal symptoms (symptomatic group) or claiming worry for possible gluten contamination in their diet even the absence of any sign or symptoms (worried group) in the previous 6 months.

\section{STAY Y 2 Questionnaire}

All patients underwent the Italian version of the State-Trait Anxiety Scales 2 (STAI Y 2) questionnaire. STAI Y 2 questionnaire deals with anxiety limited to a specific condition: in our case, the fear of gluten contamination of the food. Each question is rated on a 4-point scale not at all, somewhat, moderately so, very much so. The range of possible scores for the form Y of the STAI varies from a minimum score of 20 to 80 . STAI scores are commonly classified as "no or low anxiety" (20-37), "moderate anxiety" (38-44), and "high anxiety" (45-80). ${ }^{8}$

\section{GIP Test}

GIP test is a commercial competitive ELISA kit based on G12 monoclonal antibody (Glutendetect Biomedal, S.L., Spain). The maximum concentration of GIP urine excretion is 6-9 hours after gluten ingestion, with maximal ranges of detection of 1-35 hours. The manufacturers' declared level of detection is noted to be $2.2 \mathrm{ng} / \mathrm{mL}$ with the analytical sensitivity of GIP detection for $50 \mathrm{mg}, 500 \mathrm{mg}$ and $2000 \mathrm{mg}$ of daily gluten ingestion is $20 \%, 90 \%$ and $95 \%$, respectively. ${ }^{7,9}$ Moreover, it is recommended collecting the last urine in the night or the first in the morning if no liquid has been ingested for the last 12 hours.

Each patient included in the study received 5 GIP point-of-care tests after the presence or remote consultation. The first test was performed during the visit to train the patients. The test consists of adding a $2.5 \mathrm{~mL}$ sample of urine in the kit vial and mixed it with gentle inversions for at least 1 minute, then four drops of urine have to be added to the cell of the kit. After 15 minutes, the results are ready: one red strip means that the test is negative, the presence of the red strip plus another green strip indicates the presence in the urine of immunogenic gluten peptides. 
Patients seen in remote received the kits at home, and by email/WhatsApp, a video showing how to perform the test.

Patients were asked to perform a GIP test a maximum of 5 times in the following 5 weeks, on the occasion of worsening of the reported symptoms for the symptomatic group and/or in case of anxiety state due to the possible gluten contamination of their meals after restaurants, social events or family gathering, etc. for the worried group. They were then asked to send a picture of each test to the Clinic email.

\section{Statistical Analysis}

Categorical data were expressed as frequencies and percentages, while continuous variables as mean \pm standard deviation (mean $\pm \mathrm{SD}$ ). Possible differences between the two study groups were assessed with a $\chi 2$ test for categorical variables and an independent-samples $t$-test for continuous ones. Significance was expressed at $p<0.05$ level. The SPSS for Windows version 15.0 statistical package (SPSS Inc, Chicago, IL, United States) was used for statistical analysis.

\section{Results}

During the study period, 47 patients were considered eligible, but 17 were not enrolled for claimed occasional voluntary dietary lapses (9), IgA deficiency (1), and lack of consent to the study (7). Finally, 16 symptomatic patients and 14 patients with concerns related to gluten contamination of their diet were included. Regarding the 16 symptomatic patients, they reported gastrointestinal symptoms, such as bloating, abdominal pain, dyspepsia, and diarrhea.

Table 1 shows the characteristics of the 30 participating subjects.

The symptomatic group was composed of $11(68.8 \%)$ women and 5 males (31.2\%), while there were 11 (78.6\%) females and 3 (21.4\%) males in the worried group. There were no differences in age, BMI, compliance to GFD, and bowel habits. The worried group showed a borderline higher level of anxiety than the symptomatic group (STAI score 48.57 vs $40.31 \mathrm{p}=0.06$ ), with a significant minor percentage of patients reporting "no or low anxiety" $(14.3 \%$ vs $50 \% \mathrm{p}=0.03)$. The symptomatic patients showed a higher rate of diarrhea than group $2(25 \%$ vs $0 \%, \mathrm{p}=0.04)$.

Table I Gender, Anthropometry, and STAI 2 Scores of the Adult Celiac Patients Participating in the Study

\begin{tabular}{|c|c|c|c|c|}
\hline & Total $(\mathbf{N}=\mathbf{3 0})$ & Symptomatic Group ( $\mathrm{N}=16)$ & Anxiety Group $(\mathrm{N}=14)$ & $\mathbf{p}$ \\
\hline Gender, no. (\%) females & 22 (73.3\%) & II (68.8\%) & II (78.6\%) & 0.75 \\
\hline Mean age & $39.86 \pm 18.39$ & $35.46 \pm 16.14$ & $44.57 \pm 20.04$ & 0.18 \\
\hline BMI & $23.66 \pm 4.19$ & $24.16 \pm 3.29$ & $23.13 \pm 5.06$ & 0.5 \\
\hline Compliance (VAS 0-10) & $9.43 \pm 0.72$ & $9.5 \pm 0.73$ & $9.35 \pm 0.7$ & 0.6 \\
\hline \multicolumn{5}{|l|}{ Bowel habits } \\
\hline - Normal & $15(50 \%)$ & $6(37.5 \%)$ & $9(64.3 \%)$ & 0.14 \\
\hline - Diarrhea & $4(13.33 \%)$ & $4(25 \%)$ & 0 & 0.04 \\
\hline - Mixed & $8(26.67 \%)$ & 5 (31.25\%) & $3(21.4 \%)$ & 0.54 \\
\hline - Constipation & $3(10 \%)$ & I (6.25\%) & $2(14.3 \%)$ & 0.46 \\
\hline STAI Y2 & $44.16 \pm 12.32$ & $40.31 \pm 13.68$ & $48.57 \pm 9.13$ & 0.066 \\
\hline \multicolumn{5}{|l|}{ Anxiety state } \\
\hline - No or low anxiety & $10(33.33 \%)$ & $8(50 \%)$ & $2(14.3 \%)$ & 0.03 \\
\hline - Moderate anxiety & $2(6.67 \%)$ & 0 & $2(14.3 \%)$ & 0.11 \\
\hline - High anxiety & $18(60 \%)$ & $8(50 \%)$ & 10 (7I.4\%) & 0.23 \\
\hline GIP positive & $8(26.66 \%)$ & $5(3 \mid .3 \%)$ & 3 (21.4\%) & 0.56 \\
\hline \multicolumn{5}{|l|}{$\mathbf{N}^{\circ}$ GIP positive } \\
\hline-0 & 22 (73.33\%) & II (68.75\%) & II (78.6\%) & 0.54 \\
\hline-1 & $5(16.66 \%)$ & 3 (18.75\%) & 2 (14.3\%) & 0.74 \\
\hline-2 & $2(6.67 \%)$ & 2 (I2.5\%) & 0 & 0.17 \\
\hline-3 & I (3.33\%) & 0 & I (7.I\%) & 0.27 \\
\hline
\end{tabular}

Note: Data are expressed as percentage (\%) or as mean \pm SD. 
Gluten in urine samples was globally found in 8 out of 30 cases $(26.6 \%)$, in particular in 5/16 (31.3\%) symptomatic patients and $3 / 14(21.4 \%)$ worried ones.

\section{Real-Life Description of Selected Cases of Symptomatic Group}

Case: A 37 years old woman complaining of gastrointestinal symptoms compatible with irritable bowel syndrome (IBS) of moderate-intensity, diarrhea, and bloating predominant performed the GIP tests during flare-ups of symptoms. She reported a positive test out of 5 after eating at a social event (an office Christmas party).

However, the other tests were negative despite the flare-ups of gastrointestinal symptoms.

Case: A 49 years old man referring onset of IBS diarrhea type after a stressful life event. A complete workup was offered, including upper and lower endoscopy with histology, laboratory tests, abdominal ultrasound scan. He worked as a tour guide and was persuaded that GI symptoms were related to gluten contamination of his meals at restaurants. His work was compromised, and he agreed to undergo testing for GIP after eating out. None of the five tests was positive. He felt reassured that his symptoms were not related to his diet but a stressful life event with consequent behavioral changes.

Case: A 17 years old classic ballet dancer who received the $\mathrm{CeD}$ diagnosis in infancy. She was referred to our center because of her low body weight. She reported three positive GIP tests out of 5. After completing the observation period, she admitted voluntary daily gluten intake with the specific aim to provoke malabsorption and weight loss, to maintain the proper silhouette. After that, she was referred to psychological counseling.

\section{Real-Life Description of Selected Cases of Group 2 (Worried for Contamination)}

Cases: during the lockdown days for the COVID-19, two patients originally not included in the study called in for a remote consultation visit. They both were health-care givers with $\mathrm{CeD}$, working in COVID Hospitals. They were worried about the possible contamination of their GF meals provided, during the crisis, from the hospital canteen. We could send them three tests each, and all resulted in negative.

After completing the study, five worried patients returned to their hospital canteen without fear of the possible gluten contamination of food. One patient asked to have an additional GIP test to take with her on a trip abroad "just to stay on the safe side."

\section{Discussion}

The adherence to a strict GFD is a crucial aspect of CeD therapy. A strict and lifelong GFD ensures recovery of intestinal mucosal lesions, the disappearance of serum gluten-related antibodies, the relief of symptoms, and protection from complications. However, $\mathrm{CeD}$ patients claim a lower quality of life than controls, which can cause a low dietary compliance. ${ }^{10-12}$ A relevant percentage of adult CD patients can continue to have persistent symptoms despite a GFD. In these cases, before excluding other diagnoses (such as irritable bowel syndrome, lactose intolerant medications, etc.) or any $\mathrm{CeD}$ complications, it is essential to evaluate the gluten contamination in the diet. Patients often do not admit voluntary gluten ingestion, and sometimes they do not pay attention to what they eat. $\mathrm{CeD}$ serology can result in falsely negative and upper endoscopy can be not accepted by the patient. Therefore, a tool able to help doctors in this evaluation may be handy in clinical practice.

Our results confirm that at least 1 of $4 \mathrm{CeD}$ patients show voluntary or inadvertent GFD lapses; overall, our results are in keeping with other studies showing that almost $25 \%$ of patients in GFD had at least one positive GIP test on the stool and/or urine sample, with no differences across symptomatic and asymptomatic patients. ${ }^{6,13}$ As expected, patients worried about gluten contamination showed a borderline higher level of anxiety than the symptomatic group $(\mathrm{p}=$ 0.06 ), with a significant minor percentage of patients reporting "no or low anxiety" $(14.3 \%$ vs $50 \% \mathrm{p}=0.03)$ compared to the symptomatic group.

It is noteworthy that the available GIP detection tests on stool and urine are known to be sensitive enough to identify GIP from concentrations of $0.15 \mu \mathrm{g} \mathrm{GIP} / \mathrm{g}$ in feces and $2.2 \mathrm{ng} \mathrm{GIP} / \mathrm{mL}$ in urine, with the maximum concentration of GIP urine excretion in 6-9 hours; to take for maximum sensitivity, the manufacturers recommend taking the last urine in the night to be in the 3-15 hours window after the three main meals in the day. In a recent study, Coto et al showed that the analytical sensitivity of GIP detection for $50 \mathrm{mg}$, 500mg and $2000 \mathrm{mg}$ of daily gluten ingestion is $20 \%, 90 \%$ and $95 \%$, respectively. In the same study, the authors propose that increases in the frequency of the urine tests could improve sensibility, making the GIP multiple sampling a suitable approach to reduce false-negative results. ${ }^{9}$ 
Indeed, a negative result of both urine and stool tests was rarely observed after consuming a regular gluten-containing diet (10-20 g/day), whereas the sensibility of these tests decreased when gluten ingestion was below $50 \mathrm{mg}$. 7,14

Although our study showed a percentage of $\mathrm{CeD}$ patients with a positive GIP test consistent with the percentage of $\mathrm{CeD}$ patients with voluntary or inadvertent GFD lapses showed in literature, we cannot exclude a significant percentage of false negative due to daily gluten ingestion of less than 50mg or suboptimal time test. This concept is of clinical relevance since an adequate education of the patient about the correct timing of the test or the minimum dose of inadvertent gluten ingestion would allow obtaining a sufficient sensitivity of the GIP test.

Based on our clinical practice results, GIP can be a valuable tool to and reassure symptomatic $\mathrm{CeD}$ patients from the feeling of anxiety for gluten contamination. This tool may be of interest in dealing with teenagers or people with mental diseases or psychological problems. Moreover, verifying the gluten contamination can improve the feeling of anxiety that patients can develop in particular situations, improving their social life and, therefore, their quality of life. Another strength of the study was the GPs' involvement that offered an occasion of education on the proper follow-up of CeD patients, also in a setting different from one of a tertiary CeD dedicated clinic. During the last year, the GIP utility has been particularly evident when laboratory exams and outpatients consultations have been challenging to reach.

This study has some limitations. First, we did not perform any laboratory tests or histology to confirm GFD compliance and compare GIP tests' results. Another limitation might be the small sample size, although we have to consider the drastic reduction of outpatient visits during the COVID-19 pandemic. Last, we could not perform a more extended follow-up with GIP due to supplying difficulty of GIP tests during the early period of national lockdown; indeed, we could provide GIP tests only for 5 weeks. Further studies are needed to confirm the usefulness of the GIP test in reassuring the $\mathrm{CeD}$ patients from the worrying of gluten contamination with consequent worsening of quality of life.

In conclusion, the GIP test is a tool that can be used as a point of care test to reassure symptomatic and asymptomatic $\mathrm{CeD}$ patients from the feeling of anxiety for gluten contamination, especially useful during the COVID-19 pandemic. Further studies, including large sample populations, are required to provide evidence on the impact of the lockdown in $\mathrm{CeD}$ patients. Furthermore, GIP tests should be encouraged in celiac-devoted outpatient clinics to provide more comprehensive and effective management of these patients, such as addressing symptoms-specific therapies.

\section{Disclosure}

The authors report no conflicts of interest in this work.

\section{References}

1. Biagi F, Andrealli A, Bianchi PI, Marchese A, Klersy C, Corazza GR. A gluten-free diet score to evaluate dietary compliance in patients with coeliac disease. Br J Nutr. 2009;102(6):882-887. doi:10.1017/ S0007114509301579

2. Silvester JA, Kurada S, Szwajcer A, Kelly CP, Leffler DA, Duerksen DR. Tests for serum transglutaminase and endomysial antibodies do not detect most patients with celiac disease and persistent villous atrophy on gluten-free diets: a meta-analysis. Gastroenterology. 2017;153(3):689-701.e681. doi:10.1053/j.gastro. 2017.05.015

3. Soler M, Estevez MC, de Lourdes Moreno M, Cebolla A, Lechuga LM. Label-free SPR detection of gluten peptides in urine for non-invasive celiac disease follow-up. Biosens Bioelectron. 2016;79:158-164. doi:10.1016/j.bios.2015.11.097

4. de Lourdes Moreno M, Muñoz-Suano A, López-Casado M, Torres MI, Sousa C, Cebolla Á. Selective capture of most celiac immunogenic peptides from hydrolyzed gluten proteins. Food Chem. 2016;205:36-42. doi:10.1016/j.foodchem.2016.02.066

5. Comino I, Fernández-Bañares $F$, Esteve $M$, et al. Fecal gluten peptides reveal limitations of serological tests and food questionnaires for monitoring gluten-free diet in celiac disease patients. $\mathrm{Am}$ $J$ Gastroenterol. 2016;111(10):1456-1465. doi:10.1038/ajg.2016. 439

6. Costa AF, Sugai E, Temprano MP, et al. Gluten immunogenic peptide excretion detects dietary transgressions in treated celiac disease patients. World J Gastroenterol. 2019;25(11):1409-1420. doi:10.37 48/wjg.v25.i11.1409

7. Moreno ML, Cebolla Á, Muñoz-Suano A, et al. Detection of gluten immunogenic peptides in the urine of patients with coeliac disease reveals transgressions in the gluten-free diet and incomplete mucosal healing. Gut. 2017;66(2):250-257. doi:10.1136/gutjnl-2015-310148

8. Spielberger C. Manual for the State-Trait-Anxiety Inventory: STAI (Form Y). Palo Alto, CA: Consulting Psychologists Press; 1983.

9. Coto L, Sousa C, Cebolla A. Dynamics and considerations in the determination of the excretion of gluten immunogenic peptides in urine: individual variability at low gluten intake. Nutrients. 2021;13 (8):2624. doi:10.3390/nu13082624

10. Zingone $\mathrm{F}$, Iavarone $\mathrm{A}$, Tortora $\mathrm{R}$, et al. The Italian translation of the celiac disease-specific quality of life scale in celiac patients on gluten free diet. Dig Liver Dis. 2013;45(2):115-118. doi:10.1016/j.dld.20 12.10 .018

11. Ciacci C, Zingone F. The perceived social burden in celiac disease. Diseases. 2015;3(2):102-110. doi:10.3390/diseases3020102

12. Zysk W, Głąbska D, Guzek D. Social and emotional fears and worries influencing the quality of life of female celiac disease patients following a gluten-free diet. Nutrients. 2018;10(10):1414. doi:10.3390/nu10101414

13. Ruiz-Carnicer Á, Garzón-Benavides M, Fombuena B, et al. Negative predictive value of the repeated absence of gluten immunogenic peptides in the urine of treated celiac patients in predicting mucosal healing: new proposals for follow-up in celiac disease. Am J Clin Nutr. 2020;112(5):1240-1251. doi:10.1093/ajen/nqaa188

14. Comino I, Real A, Vivas S, et al. Monitoring of gluten-free diet compliance in celiac patients by assessment of gliadin 33-mer equivalent epitopes in feces. Am J Clin Nutr. 2012;95(3):670-677. doi:10.3945/ajcn.111.026708 


\section{Publish your work in this journal}

Clinical and Experimental Gastroenterology is an international, peerreviewed, open access, online journal publishing original research, reports, editorials, reviews and commentaries on all aspects of gastroenterology in the clinic and laboratory. This journal is indexed on American Chemical Society's Chemical Abstracts Service (CAS).
The manuscript management system is completely online and includes a very quick and fair peer-review system, which is all easy to use. Visit http://www.dovepress.com/testimonials.php to read real quotes from published authors. 